\title{
Polydisperse Packings
}

\author{
H.J. Herrmann, R. Mahmoodi Baram, and M. Wackenhut \\ Institute for Computer Applications 1, University of Stuttgart, Pfaffenwaldring 27, D-70569 Stuttgart
}

Received on 23 April, 2003

\begin{abstract}
The manufacture of high resistance concrete or hard ceramics needs extremely dense granular packings. They can only be realised when the size distribution of grains is strongly polydisperse. Typically powerlaw distributions give the best results. We present a simple packing model for polydisperse distributions, namely a generalized reversible parking lot model. We also discuss the perfectly dense limit, namely Apollonian packings in three dimensions and show in particular the existence of space filling bearings rotating without slip and without torsion.
\end{abstract}

\section{Introduction}

The search for the perfect packing has a long history [1] and although much is known about monodisperse or bidisperse systems, the real challenge lies in polydispersity. Materials of very high resistance made of an originally granular mixture as it is the case for high performance concrete (HPC) [2] and for hard ceramics are manufactured by trying to reach the highest possible densities. From the fracture mechanics point of view, higher densities imply less and smaller microcracks and therefore higher resistance and reliability. This goal can be reached as shown clearly for the case of HPC by mixing grains of very different sizes (gravel, sand, ordinary cement, limestone filler, silica fume), where the size distribution of the mixture follows as closely as possible a powerlaw distribution. In fact it is known that configurations of density one are obtained for spherical particles in so-called Apollonian packings (albeit not yet physically realisable) and constitute the idealized final goal of a completely space filling packing having absolutely no defects.

In the studies presented here, on the one hand we generalize a toy model for granular compaction, namely the reversible parking lot model to size distributions following a powerlaw. On the other hand we will discuss possible different realisations and self similar packings in three dimensions.

\section{A reversible parking lot model for polydisperse size distributions}

Parking lot models have served as simple representations of compaction phenomena. Ben-Naim and Krapivski [3] introduced a reversible parking lot model to describe the compaction dynamics of monodisperse packings. They found an asymptotically logarithmic approach to a final density which was confirmed experimentally by Knight et al. [4]. The model is defined within a one-dimensional interval on which particles of fixed size are randomly absorbed with a rate $k^{+}$and desorbed with a rate $k^{-}$. The density reached after an infinite time depends on the ratio $k^{-} / k^{+}$and is unity when this ratio vanishes.

For strongly polydisperse size distributions, this model must be considerably modified in order to still make sense $[5]$.

- A finite reservoir of particles must be considered in order to keep the distributions the same and this reservoir must be essentially not larger than the actual particles one would need to fill the interval.

- The system must be initialized very carefully by putting first the large particles, otherwise small particles will create huge voids.

- A size dependent desorption probability must be considered, otherwise the large particles will easily leave the system without being able to be reinserted.

Our model is defined in the following way: Let $r_{i}$ be the diameter of the ith-particle. Then the reservoir is filled with $K$ particles following a distribution proportional to $r_{i}^{-b}$ for $r_{i} \in\left[r_{\min }, r_{\max }\right]$ and fulfilling the constraint $\sum_{i}^{K} r_{i}=l$ where $l$ is the length of the interval. The system is initialized by inserting the particles according to their size, starting with the largest one. Each particle gets $I$ attempts to find a free space in the interval and when it does, it will be left there. If $I$ is large enough, most particles will actually already be placed in this initial state. Once this procedure is finished, i.e. all particles in the reservoir have had their $I$ attempts, the real compaction dynamics is switched on by choosing randomly one of the remaining particles from the reservoir and attempting to absorb it and then choosing randomly a particle in the interval with probability $p(r)$ in order to desorb it. Each such step is called a time unit and typically we perform $t=10^{9}$ such units. The desorption probability 
is defined through

$$
p(r)=\sum_{i=1}^{K_{1}} '\left(h_{i}-r\right) / l
$$

where $K_{1}$ is the number of particles on the interval, $h_{i}$ the size of the ith-hole and the prime at the sum denotes that the sum only goes over positive terms and the negative ones are discarded.

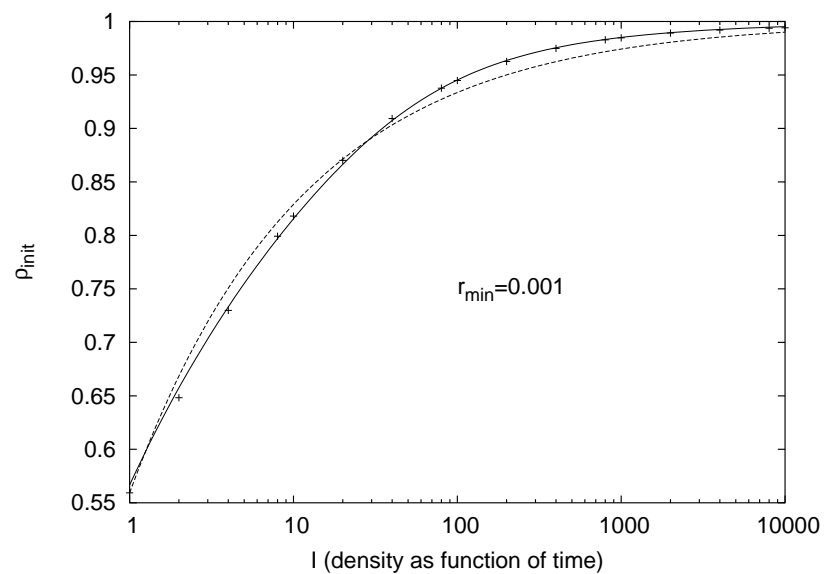

Figure 1. Density as function of time.

In Fig. 1 we see an example for the evolution of the density as function of time. The first part with the steepest increase corresponds to the initialization (up to $t=10^{7}$ ) and the density reached at this point is called $\rho_{\text {init }}$. From there we continue using the dynamics with desorption plotting the data along the same time scale until $t=10^{9}$. The full line is a fit using the equation

$$
\rho_{\text {init }}(I)=\rho_{\max }-\frac{\Delta \rho}{1+B \cdot \ln (1+I / \tau)} I^{-f_{n}}
$$

while the dotted line is obtained when the quotient in Eq. (2) is removed, $\Delta \rho, B, \tau$ and $f_{n}$ are essentially fit parameters. One sees that on a logarithmic time scale eventually densities close to unity can be obtained. A particularly interesting result of this model is presented in Fig. 2 where the finally reached density at fixed $I$ is shown as a function of the exponent of the powerlaw size distribution. We see that there exists an optimal value for $b$ around 1.6. Generalising the above model to higher dimensions should therefore be a way to help designers of stronger materials optimizing the size distribution of the grain mixture.

\section{Space filling packings}

One ultimately wants to reach a packing of density one. Well known configurations are so-called Apollonian packings in which a sphere is always placed inside an existing pore such as to have maximum volume. The new smaller pores created by this procedure are then again filled with even smaller

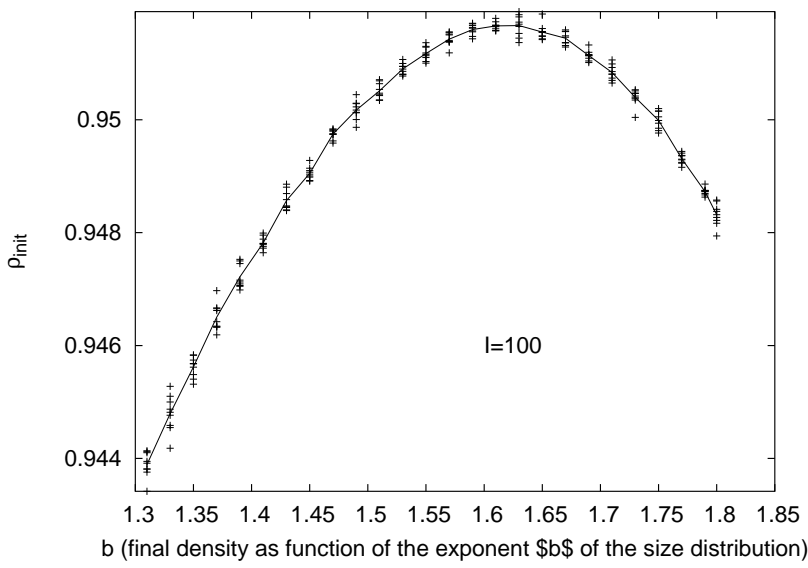

Figure 2. Density after the initialization as function of the exponent $b$ of the size distribution.

spheres of maximum volume and so on. If one starts with mutually touching spheres on the vertices of a tetrahedron, one obtains the classical Apollonian packing [6, 7] which is known to be self-similar, its size distribution is a perfect power law with exponent 3.47 and from there results that the pore volume and the overall surface are fractal with a fractal dimension of 2.47 .

We found four more such self-similar configurations with different topologies and therefore possibly different exponents, one based on the cube, two on the octahedron and one on the dodecahedron and at this point no further selfsimilar packings are known [8].

The biggest surprise however is that one of those configurations, namely one based on the octahedronal symmetry is bi-chromatic, this means that two colours suffice to assure that spheres having the same colour never touch. This is shown in Fig. 3. Its fractal dimension is about 2.54, i.e. larger than the classical Apollonian one. That means that a blue sphere only has red neighbours and vice-versa. Furthermore we can prove [9] that if one chooses any axis and rotates one sphere around it, all the other spheres will roll without slip at their contacts. The rotation axis of the different spheres are then in general different. If the blue ones rotate clockwise, the red ones rotate counterclockwise. One can also show that except for a set of points of measure 0 , the contacts have no torsion friction, i.e. that the two spheres in contact will have equal angle of velocities of opposite sign in the axis perpendicular to the tangent plane. Therefore one has essentially only rolling friction which typically is very small so that this packing is a realisation for a threedimensional space filling bearing, actually to our knowledge the first one ever described.

\section{Conclusion}

The highest possible densities are reached by polydisperse granular packings. We have presented a simple onedimensional model, showing that one can logarithmically 


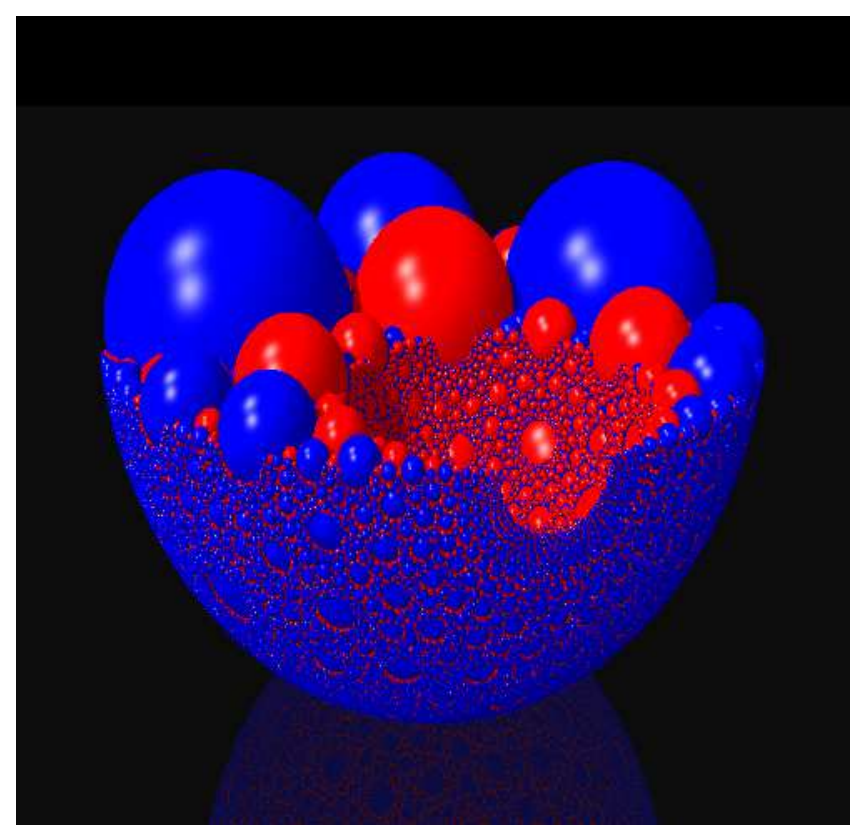

Figure 3. Bi-chromatic packing of fractal dimensions 2.59.

slowly attain densities above $95 \%$ and that one can optimize this number by tuning the exponent of the distribution. We have also seen that the idealized completely dense case can be self-similar in five topologically different configurations, only one of them having the property of being a bearing, i.e. allowing for slipless rotations around an arbitrary axis.

A full three-dimensional model or simulation of a system is still far from being realised because of the difficulties to deal with the large amount of very small particles. Our contributions are just a small step in this direction. From the theoretical point of view one has also to consider non-selfsimilar perfect packings and non spherical particles, understand the settling and demixing dynamics and calculate for each size the corresponding mobilities. From a numerical point of view, one has to organize the data hierarchically, eventually using quad-trees for a generalized linked cell algorithm and consider size classes in representative volume elements. We think that in the future much progress can still be achieved.

\section{References}

[1] T. Aste and D. Weaire, The pursuit of perfect packing, Institute of Physics Publishing, Bristol and Philadelphia 2000.

[2] F. de Larrard, Concrete Mixture Proportioning, Eds. E \& FN Spon, London, New York 1999.

[3] E.R. Nowak, J.B. Knight, E. Ben-Naim, H.M. Jäger, and S.R. Nagel, Physical Review E, 57 (2), 1971 (1998).

[4] J.B. Knight, C.G. Frandich, Chun Ning Lau, H.M. Jäger, and S.R. Nagel, Physical Review E, 51 (1), 3957 (1995).

[5] M. Wackenhut and H.J. Herrmann, Searching for the perfect packing. Preprint, 2003.

[6] D.W. Boyd, Math. Comp. 27 (122), 369 (1973).

[7] M. Borkovec, W. de Paris, and R. Peikert, Fractals 2 (4), 521 (1994).

[8] R. Mahmoodi Baram and H.J. Herrmann, Self-similar packings in three dimensions. Preprint, 2003.

[9] R. Mahmoodi Baram, H.J. Herrmann, and N. Rivier, Space filling bearing in three dimensions. Preprint, 2003. 